\title{
Quantitative kinetics and enthalpy measurements of biphasic underflow chemical reactions by InfraRed Thermography
}

\author{
M. Romano ${ }^{1, a}$, C. Pradere ${ }^{2, b}$, J. Toutain $2, c$,J.C. Batsale ${ }^{2, d}$ \\ 1 LOF, University Bordeaux 1, UMR CNRS-Rhodia-UB1 5258, 178, Avenue du docteur Schweitzer, 33608 Pessac, France,. \\ 2I2M, Department TREFLE, UMR CNRS 5295 - site ENSAM Esplanade des Arts et Métiers, 33405 Talence Cedex, France \\ a romano@enscbp, bc.pradere@i2m.u-bordeaux1.fr, c toutain@enscbp, djc.batsale@i2m.u-bordeaux1.fr
}

Keywords: chemical reaction, microfluidics, heat transfer, calorimetry, biphasic flow, hydrodynamics.

\begin{abstract}
The scope of this paper is to present the experimental study of a well known chemical reaction in a biphasic millifluidic droplet flow by using InfraRed Thermography. This simple thermal evaluation enables the characterization of kinetics and enthalpy of exothermic chemical reactions. The originality of this work is the application of a very simple thermal model based on an homogenized thin body approximation to perform calorimetric estimations. This novel calorimeter needs a thermal calibration step to estimate the heat losses (W). Then, a correlation method is applied for the simultaneous estimation of the heat source $(\varphi)$ and the characteristic coefficient due the convective effects $(H)$. Here the estimation of the characteristic coefficient $\mathrm{H}\left(\mathrm{s}^{-1}\right)$ is done at each flow rate ratio $(\mathrm{R})$. This procedure is applied for several chemical reaction performed at different flow rate ratios. Then, the enthalpy is estimated with an error lower than $2 \%$. In addition, the methodology to estimate the mixing kinetics of the reaction can be pointed out by the integrated flux over the time. Finally, a non contact thermal calorimeter based on millifluidic and IR thermography was developed. It is a convenient and powerful tool for the characterization of chemical reaction performed in a droplet flow.
\end{abstract}

\section{Introduction}

Micro and millifluidic droplet based systems are recognized techniques for high-throughput screening platforms. Chemistry uses reactive droplet flows platforms as a tool for analysis, process intensification and data acquisition [1]. Thus, InfraRed Thermography (TIR) is a versatile technology that can be applied in a very wide range of domains and scale going from macroscopic applications to the observation of miniaturized systems such as electronic devices characterization or biological and chemical systems. Indeed, the principal motivation for the development of a non intrusive tool for online chemical reactions monitoring at small scale is : to profit from the combination of both flexible and original techniques. More precisely, quantitative IRT studies offer the possibility to measure important experimental parameters as the convective heat transfers [2] but also to monitor the temperature distribution, which could be one of the most important parameter in some experimental studies, as is the case of chemical reactions characterization in terms of kinetics and thermodynamics. However, many chemical or physical processes are associated with thermal phenomena commonly characterized by calorimetric methods to obtain thermodynamic data and kinetics by measuring the heat flux. Where the evolution of heat flux is reproducible and directly proportional to the reaction conversion rate. Among them, some in-situ calorimetric applications have been developed for both miniaturized and flow-based systems. However, despite their promise most of techniques are intrusive, difficult to adapt and have been tested on single phase flows only. Hence, these techniques are not suitable to be used as a flexible online analysis tools. From this point of view, the aim of this work remains on the development of simple thermal evaluation device designed for microfluidic droplet flows. In fact, through successive steps the estimation of the heat source term acting inside the droplet will be done. 


\section{Experimental set up and materials}

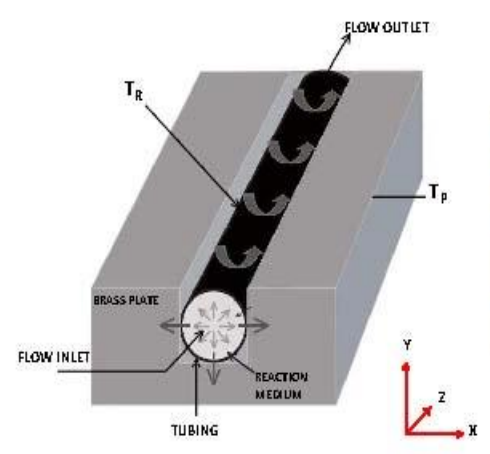

A

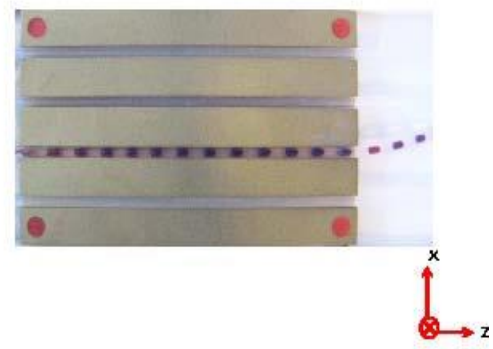

B

Fig.1. A) Scheme of the isoperibolic system. B) Millifluidic droplet based system, is composed of a brass chip and a TEFLON tubing.

The millifluidic isoperibolic chip is schematized on Fig.1, it is designed by using a brass bulk for thermal control whereas the flow set-up is realized inside small-size commercial TEFLON tubing and junctions. The typical dimensions of the main tubing are $3.17 \mathrm{~mm}$ (outer) $1.6 \mathrm{~mm}$ (inner) diameter. The tubes used for supplying the reactants have $500 \mu \mathrm{m}$ (outer) and $250 \mu \mathrm{m}$ (inner) diameter. This design allows to master the initial and the boundaries conditions. The brass bulk is thermally regulated by a Peltier module from -5 to $90 \circ \mathrm{C}$ for an accurate control cooling or heating of the tubes, the boundary condition of the external diameter of the tubing is isoperibolic (i.e. imposed temperature). As a consequence, the temperature inside the tubing (reaction media) results of the heat transfer coefficient between the imposed temperature of the brass bulk and the inner diameter of the tube. Fig. 1.A shows a cross-sectional representation of the isoperibolic system, it can be noted that $3 / 4$ of the tube are in contact with the brass plate. This induces that the heat transfer acts as a thermal conductance as it was previously published [3]. Inside the reactor (tubing), the biphasic flow is performed by a high precision syringe pump NEMESYS from Cetoni. This device is suitable for high precision delivery of micro volumes, thus droplets are generated by the injection of the fluorinated IKV oil, v= $32 \mathrm{cSt}$ at $25 \circ \mathrm{C}$ (continuous phase) and the reactive phase at different flow rate ratios. As a consequence the hydrodynamics parameters are under control, such as: the total flow rate (i.e. droplet velocity), the droplet size and the volume fraction of oil and droplets. Here a chemical reaction between hydrochloric acid $(\mathrm{HCl}, \mathrm{Sigma}$ Aldrich) and sodium hydroxide base (NaOH Sigma Aldrich) was performed at a total flow of $\mathrm{Q}_{T}=10 \mathrm{~mL} . \mathrm{h}^{-1}$. The two reagents were added into the droplets at the same initial concentration $\left(C_{0}=0.5 \mathrm{M}\right)$. The total flow of the droplet is equal to the sum of both reagents flows $Q_{D}=Q_{a c i d}+Q_{b a s e}$. and the oil flow $\left(Q_{O}\right)$ was imposed at different flow rate ratios $\left(R=Q_{O} / Q_{D}\right)$ summarized in Table

An infrared FLIR camera model SC7000 is used for the temperature field measurements, built up 76800 individual InSb detectors, with an optimum wavelength between 2.5 to $5.2 \mu \mathrm{m}$, a pitch of $30 \mu \mathrm{m}$. The IR objective lens is a MWIR F/2 of 25 $\mathrm{mm}$. The spatial resolution of the temperature measured by each pixel of the sensor is about $250 \mu \mathrm{m}$. The scanning frequency is up to $1 \mathrm{kHz}$.

Table 1: Experimental droplet-oil flow rate ratios

\begin{tabular}{|c|c|c|}
\hline $\mathrm{R}=\mathrm{Q}_{\mathrm{O}} / \mathrm{Q}_{\mathrm{D}}$ & Oil flow $\mathrm{Q}_{\circ},\left(\mathrm{mL} \cdot \mathrm{h}^{-1}\right)$ & Droplet flow $\mathrm{Q}_{\mathrm{D}},\left(\mathrm{mL} \cdot \mathrm{h}^{-1}\right)$ \\
\hline 0.5 & 3.33 & 6.66 \\
\hline 2 & 6.67 & 3.33 \\
\hline 4 & 8 & 2 \\
\hline 8 & 8.89 & 1.11 \\
\hline 10 & 9.09 & 0.91 \\
\hline
\end{tabular}

\section{Principle of the proposed method}

A chemical reaction is performed inside the droplet, here each droplet can be assimilated to an isolated chemical reactor. Besides, the biphasic flow is analyzed by InfraRed (IR) thermal measurements. The different temperature fields are reported to show that the spatial evolution of the temperature is periodic (Fig.2). It is important to note that the temperature is measured in arbitrary unities, called Digital Level (DL). Fig.2 illustrates, the thermal phenomena can be managed according different orders, in fact the profiles exhibit that the total temperature can be decomposed according to two contributions: (i) continuous one (Fig 2.B) and (ii) fluctuated one (Fig. 2.C)

$$
T\left(z, t_{i}\right)=\mathrm{T}(\mathrm{z})+\tilde{\mathrm{T}}\left(\mathrm{z}, \mathrm{t}_{\mathrm{i}}\right)
$$

It is important to precise that the Continuous Contribution (CC) schematized on Fig.2.B, represents almost $90 \%$ of the signal. While the fluctuated component highlights the presence of the biphasic flow as shown on Fig 2.C, but represents less than $10 \%$ of the temperature profile at a time $i$. The Biot number of the droplets is lower than 1 , so the temperature homogeneity in the $r$ direction allowed to neglect the heat transfer along this direction. Moreover, it is important to note that the velocity field 
within the droplet evidence recirculations, while the velocity field within the oil phase revealed velocity fields closed to a plug flow (or piston flow) [4]. Then, the recirculation inside the droplet also enhances the temperature homogeneity. Finally, from all these assumptions a thin body media model can be used to approximate the global heat transfer in such millifluidic and isoperibolic system. More precisely, in this approximation, only the parietal heat exchanges between the equivalent homogeneous medium and the brass bulk will be taken into account, the model is expressed as follow:

$$
\left(\rho C_{p} \mathrm{~V}\right)^{*} \frac{\mathrm{d}_{\mathrm{CC}}(t)}{d t}=\frac{\phi(\mathrm{t})}{(1+\mathrm{R})}-\mathrm{h}_{\mathrm{p}} \mathrm{S}\left(\overline{\mathrm{T}_{\mathrm{CC}}(t)}-T_{p}\right)
$$

Where $\varphi(t)$ represents the heat source $(W), h_{p}\left(W \cdot m^{-2} \cdot D L^{-1}\right)$ the parietal heat exchange coefficient between the tubing and the isoperibolic boundary, $S$ is the heat exchange surface $\left(S=\pi d L T, m^{2}\right),\left(\rho C_{P} V\right) *$ defines the equivalent homogeneous medium composed by droplet and the oil phase, denoted by the suffixes $D$ and $O$ respectively. Thus Eq. 2 can be rewritten as:

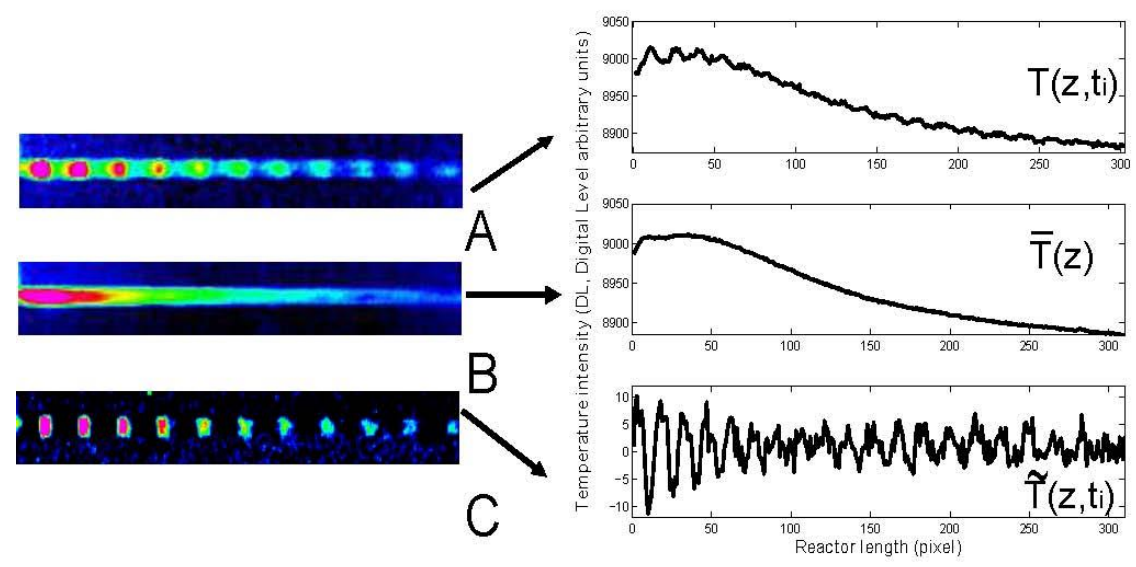

Fig. 2 Thermal phenomena at different orders: A). IR image : droplet flow temperature field at time t with a chemical reaction.

B) IR averaged image of the temperature field over $\mathrm{N}$ periods. C) IR temperature field of fluctuated component. Right side:

A). Temperature profile along the channel at time $t\left(T\left(z, t_{i}\right)\right)$. B). Temperature profile along the channel where the temperature field is averaged over $N$ periods $C$ ). Fluctuated profile along the channel $\tilde{T}\left(z, t_{i}\right)$ this signal is also equal to the subtractions of signal $B$ and $A$.

$$
\frac{\mathrm{dT}_{\mathrm{CC}}(t)}{d t}=\frac{H \phi(\mathrm{t})}{\mathrm{W}(1+\mathrm{R})}-\mathrm{H}\left(\overline{\mathrm{T}_{\mathrm{CC}}(t)}-T_{p}\right)
$$

The variables are summarized as:

$$
W=h_{p} \pi \mathrm{dL}_{\mathrm{T}} ; \mathrm{H}=\frac{\mathrm{W}}{\left(\rho \mathrm{C}_{\mathrm{p}} V\right)^{*}} \text { and } \Phi=\frac{H \phi(\mathrm{t})}{\mathrm{W}}
$$

In order to estimate the heat source $\varphi$ (i.e. enthalpy of the chemical reaction), the $\mathrm{H}$ and $\mathrm{W}$ coefficients are experimentally estimated. This last estimation implies the calibration procedure, which is necessary to perform an accurate and qualitative estimation from the experimental data. The $\mathrm{H}$ coefficient represents the inverse of the characteristic time $\left(\mathrm{s}^{-1}\right)$. W coefficient represents the parietal heat losses due to the isoperibolic condition imposed by the brass bulk (W.DL $\left.{ }^{-1}\right)$.

\section{Results and discussions}

The $\mathrm{W}$ coefficient represents the parietal heat losses due to the isoperibolic condition imposed by the brass bulk (W.DL $\left.{ }^{-1}\right)$. This last calibration allows to link the heat source dissipated inside the tubing and the temperature measured at its surface. For that purpose, an electrical conductive tin wire is introduced inside the tubing channel (this results are not shown). The correlation method is applied for the simultaneous estimations of the heat source and characteristic time due the convective effects [5]. The enthalpy of the reaction (i.e. heat source) implies a difficult estimation, because in such situation, the source term evolution is correlated with heat losses. An acid-base chemical reaction with a well known enthalpy is performed. The estimated spatial or temporal distribution of the heat source do not correspond to the kinetic of the reaction. In such a case, what is call kinetics is more a gradual mixing of the chemical products due to mass diffusion. 


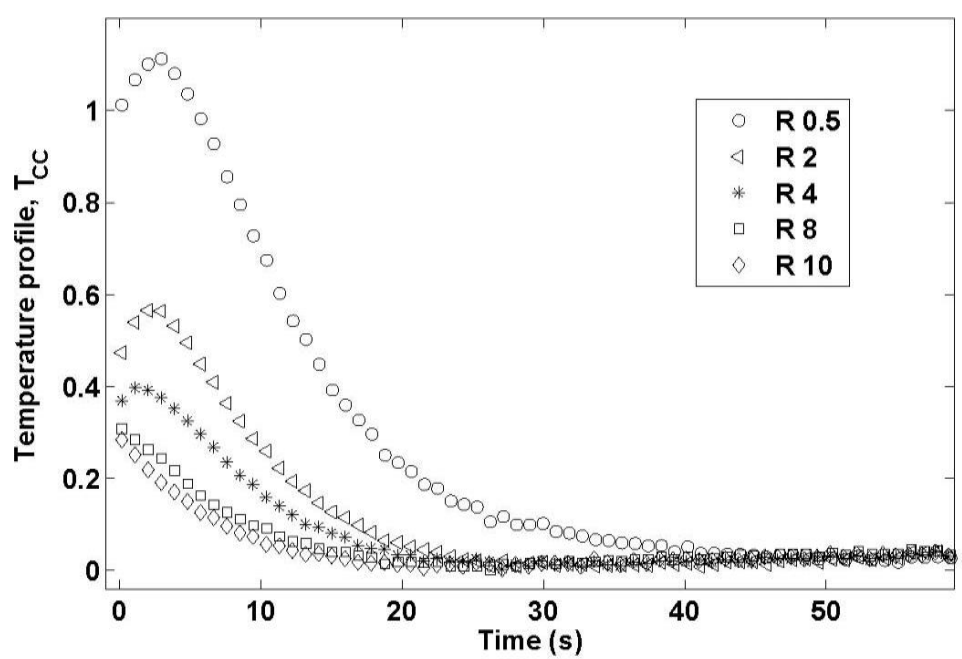

Fig. 5 Measured Continuous Contribution (CC) for a given total flow rate $10 \mathrm{~mL} \cdot \mathrm{h}^{-1}$ of an acid base chemical reaction at different flow rate ratios summarized on Table 1 at the room temperature. Note: Temperature profiles of the CC are measured in Digital level (DL) an arbitrary unit.

The temperature profiles of the chemical reaction are described according to Eq.3 in Fig.5, the continuous contribution for different flow rate ratios $(R)$ is illustrated. The temperature profile intensity $(T, D L)$ of the acid-base reaction tends to decrease as the $\mathrm{R}$ is increased. Indeed, when the droplet-oil ratio is weak (ex. $\mathrm{R}=0.5$ ) the relaxation time is higher and proportional to a higher molar flow; as the ratio increases (until $\mathrm{R}=10$ ) the molar flow decreases and the biphasic flow reaches earlier the temperature of the isoperibolic chip. It is important to note that the temperature profiles for the ratios $\mathrm{R}=8$ and 10 are almost the same because the volume of the droplet is almost the same as well.

In order to remove the convective effects information, it is important to detect the time during the one only the heat source or where the convective effects are estimable. From the knowledge of the exact zone where the heat source is OFF, it becomes possible to estimate the $\mathrm{H}$ coefficient as function of time. Or when the heat source is $\mathrm{ON}$, it is possible to estimate the heat source by applying Eq. 5. Then, by applying the Eq.6 the integral of the estimated heat source over the time is estimated.

$\phi(t)=\left[\frac{\mathrm{d} \overline{\mathrm{T}_{\mathrm{CC}}(t)}}{d t}+\mathrm{H}\left(\overline{\mathrm{T}_{\mathrm{CC}}(t)}-T_{p}\right)\right]+\frac{\mathrm{W}(1+\mathrm{R})}{\mathrm{H}}$

$\Phi=\int_{t=0}^{t=e n d} \phi(t) d t=M \Delta H \quad$ where $\quad \mathrm{M}=\frac{\mathrm{Q}_{\lim } C_{0} t_{\text {res }}}{2}$

$M$ represents the injected concentration, $C_{0}$ is the initial concentration of the limiting reactant (mol. $\left.L^{-1}\right)$, $Q_{\lim }$ is the limiting reactive flow $\left(\right.$ L. $\left.^{-1}\right)$ and tres the residence time $(\mathrm{s})$. In this equation $\mathrm{M}$ is divided by 2 , because the reactants flows and the initial concentrations are the same.
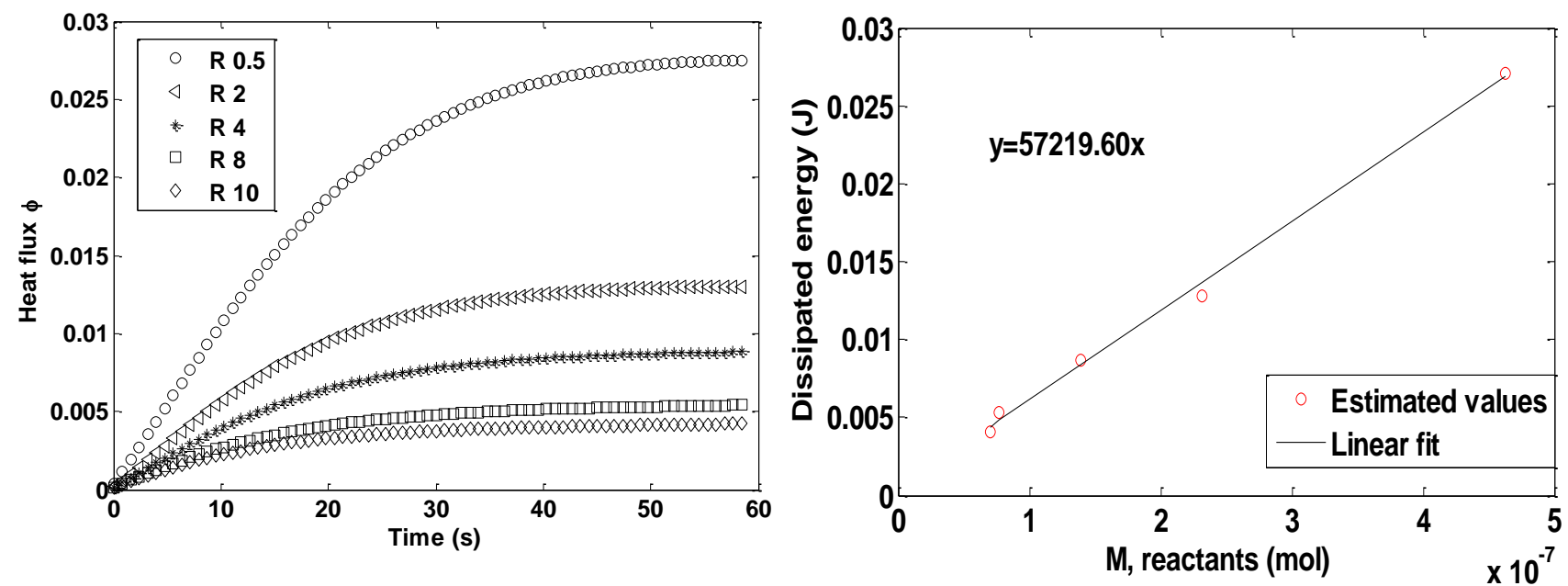
Fig. 6 A) Dissipated heat source of the acid-base chemical reaction along the channel during the residence time. B) Estimated heat source for the acid base reaction at a total flow of $10 \mathrm{~mL} \cdot \mathrm{h}^{-1}$ for different droplets-oil flow ratios (summarized on table 1) as a function of $\mathrm{M}$ the injected reactants.

From the estimation of the heat source over the time on Fig. 6.A and by applying the Eq. 6 the total heat source dissipated by the chemical reaction is characterized by integration of the heat source $\Phi(\mathrm{W})$. In this figure, it can be observed that the intensity of the dissipated heat sources tends to decrease as the ratio is increased, this is because the molar flow of the reagents also decreases. It can be also observed that once the maximum heat source has been dissipated, the source remains constant over the channel. In addition, the plateau is not reached at the same time for all the $\mathrm{R}$ ratios, this may be a signature of the mixing time. In order to make an accurate estimation of the enthalpy, the reaction has to be complete (fully mixed). This is because the integration of the heat source when the conversion is total inside the channel provides direct access to the reaction enthalpy. The estimation of the enthalpy $\Delta \mathrm{H}$ is done by plotting the integrated heat source $\Phi$ versus the concentration $\mathrm{M}\left(\mathrm{Q}_{\mathrm{lim} .} \mathrm{C}_{0} . \mathrm{tres}_{\mathrm{res}}=\mathrm{mol}\right)$. Fig. 6.B shows the integrated heat source as a function of $\mathrm{M}$, the concentration of the injected reactants (proportional to the molar flow rate). From this data, the enthalpy of the reaction (mixing in this case) can be estimated through a linear regression analysis. The slope represents the estimated enthalpy, the obtained value is 57.22 $\mathrm{kJ} . \mathrm{mol}^{-1}$ which is in good agreement with the value given in the literature $56 \mathrm{~kJ}^{\mathrm{mol}}{ }^{-1}[6]$, the estimation exhibits less than $2 \%$ of error. The uncertainties of the repeatability are acceptable, and the experimental repeatability for the enthalpy $(\Delta \mathrm{H})$ estimation is approximately $94 \%$, implying that this parameter is accurately estimated.

\section{Conclusions}

A non contact calorimeter, composed by an IR camera and a isoperibolic brass chip was developed and validated for reactive two phase flows characterization. In this case the acid $(\mathrm{HCl})$ base $(\mathrm{NaOH})$ reaction was studied. A thin body media model was used to approximate the global heat transfer in such millifluidic system. In this approximation, only the parietal heat exchange between the equivalent homogeneous medium and the brass bulk are taken into account. Thus, in order to perform a quantitative estimation of the heat source $\varphi$ (i.e. enthalpy of the chemical reaction), the $\mathrm{H}$ and $\mathrm{W}$ coefficients were experimentally estimated. The $\mathrm{W}$ coefficient represents the parietal heat losses due to the isoperibolic condition imposed by the brass bulk. The correlation method was used for the simultaneous estimation of the heat source $(\varphi)$ and the characteristic time due the convective effects $(H)$. This procedure was perform for each the chemical reaction. In addition the heat source was estimated of the acid-base enthalpy estimation with less than $2 \%$ error. The acid-base reaction was limitated by the mass diffusion phenomenon, which is a signature of the advancement of the reaction. This work shows that there is no obstacle to estimate heat source and kinetics in such biphasic flow by applying the proposed methodology including the thermal calibration. Finally, IR thermography is a convenient and powerful tool for the characterization of highly exothermic chemical reactions.

\section{References}

[1] H. Song, J. D. Tice, and R. F. Ismagilov, "A microfluidic system for controlling reaction networks in time," Angewandte Chemie, vol. 115, no. 7, pp. 792-796, 2003.

[2] Y. Rouizi, D. Maillet, Y. Jannot, and I. Perry, "Inverse convection in a flat mini-channel: towards estimation of fluid bulk temperature distribution with infrared thermography," in Journal of Physics: Conference Series, vol. 395

[3] C. Hany, C. Pradere, J. Toutain, and J. Batsale, "A millifluidic calorimeter with infrared thermography for the measurement of chemical reaction enthalpy and kinetics," QIRT, vol. 5, no. 2, pp. 211-219, 2008.

[4] F. Sarrazin, K. Loubiere, L. Prat, C. Gourdon, T. Bonometti, and J. Magnaudet, "Experimental and numerical study of droplets hydrodynamics in microchannel," AIChE Journal, vol. 52, no. 12, pp. 4061-4070, 2006.

[5] C. Ravey, C. Pradere, and J. C. Batsale, "Heat transfer and correlation mapping for the estimation of thermophysical properties in microfluidic devices," ASME, 2010.

[6] Lidie. D.R., ed., Handbook of Chemistry and Physics. Ohio: CRC Press, 72 ed.,1992. 